\title{
Salesperson Compensation Contract Design Based on Multi-agent Principal Agent Model
}

\author{
Hongyan Liu \\ ${ }^{1}$ Economics and Management Department, North China Electric Power University, Baoding City, \\ Hebei Province, China
}

Keywords: Compensation incentive, Multi-agent principal agent model, Sales system

\begin{abstract}
The effective and efficient motivation of the sales personnel affects the sales of a firm directly. This paper studies the incentive effects of different compensation contracts under the framework of multi-agent principal agent model, and finds that the optimal contract is not the one that ties one salesperson's compensation to his own performance, but the one that ties his compensation to all the salespersons' performance. Factors that influence the incentive degree are also discussed.
\end{abstract}

\section{INTRODUCTION}

It is very important for firms to motivate the salesmen effectively and efficiently. Sales personnel may have moral risk behaviors, such as giving customer orders to rival, reporting false information, exaggerating competition degree, concealing market capacity, etc. Two ways can be used to effectively guard against their moral hazard problem. One is to establish a reliable information sharing system, which can alleviate information asymmetry and which is restricted by factors like the cost and the sharing willingness of related parties; the other is an effective compensation incentive mechanism.

Nowadays multi-agent principal-agent model has been widely applied to many fields to study compensation incentive problems. For example, Sunil Dutta and Regina M. Anetil(1999) analyzed the interrelationship between two divisions when there are internal transfer prices between the two divisions a . Zhi Xiaoqiang (2005) studied, using multi-agent principal-agent model, the controllability principal in divisional manager's performance evaluation and the factors that influence the incentive coefficient in an incentive contract ${ }^{\mathrm{b}}$. Tian Houping et al (2007) proposed the pay scheme designing method is proposed with Multi-Agent Competition on the assumption that the market demand is sensitive to effort level and the two sellers have competition, They find that the agents have to improve their efforts with the increasing of competition, and they prefer to lower risk sharing and fixed wages when there is more uncertainty of market and costs of effort, and the principal may benefit by making use of the agents' competition ${ }^{c}$. Tian Ying et al (2006) find that the more important task applied to the stronger incentive contract. Similarity, the stronger incentive contract applied to the more uncertain task. The more replacement there is between the two tasks, 
the stronger the incentive contract should give to the task which is more important or uncertain ${ }^{\mathrm{d}}$. Lin Yinghui and Tu Meizeng (2005) set up a game model for the incentive contract design. They find that in order to make supply chain coordination effective, the core enterprise should provide different contracts instead of the same contract to the agents ${ }^{\mathrm{e}}$.

This paper studies the incentive effects of different compensation contracts under the framework of multi-agent principal agent model.

\section{Model}

Suppose a company has two sales persons (or two sales stores) whose efforts cannot be observed. The company, as the principal, links the compensation of the agents to their performances to induce a higher level of effort. Suppose there is a competition relationship between the two agents (such as two nearby stores selling some partially or completely replace goods). The company and the agents (salesmen) are both to maximize their own expected utility. Assume that $\pi_{i}$ represents the performance of salesperson $i,(i=1,2)$, and:

$$
\pi_{i}=\sum_{j=1}^{2} g_{i j} a_{j}+\theta_{i},(i=1,2) \cdot
$$

Where $\mathrm{a}_{\mathrm{i}}$ represents the effort level of salesperson $i$. Further, $g_{i j}$ is the marginal effect of the salesperson $j$ 's effort on the sales volume of the company, and $g_{i j}<0$. This means that effort of the salesman $j$ have a negative impact on the sales of salesperson i. also, gij reflects the degree of competition between the two agents, the bigger the value of $\left|g_{i j}\right|$, the higher the degree of competition. This can be considered as that, when their opponents' (or their colleague's) effort level is constant, increasing their own effort level can attract over the opponent's (or the colleague's) customers. Assume $g_{i i}>0$ and $g_{i i}>\left|g_{i j}\right|$, which means the impact of salesperson i's effort on his own performance is greater than that of his colleague's. $\vartheta_{i}$ is a normally distributed random variable which represents the external environment, with $\vartheta_{i} \in\left(0, \sigma_{i}^{2}\right)$, and $\operatorname{Covariance} \operatorname{cov}\left(\vartheta_{1}, \vartheta_{2}\right)=0$. In order to simplify the problem, assume that the volatility of the two sales personnel performances are the same, that is, $\vartheta_{1}=\vartheta_{2}=\vartheta$.

Assume the principal is risk neutral, and the agent is risk averse. The performance of the company (or the sales volume) is $\pi=\pi_{1}+\pi_{2}$ and the objective of the principal is to maximize the performance of the company. Suppose the agents' utility function is $U=e^{-\rho \omega}$, where $\rho$ is the agent's absolute risk coefficient, and $\varpi$ is the monetary income the agent derives from the compensation. The agent maximizing his expected utility is equivalent to maximize his certainty equivalent, $Y_{i}=E\left(S_{i}\right)-\frac{1}{2} \rho \operatorname{var}\left(S_{i}\right)-C_{i}\left(a_{i}\right)$, where $S_{i}$ is the salesperson i's compensation, $C_{i}\left(a_{i}\right)$ is the cost of salesperson i's effort, $\mathrm{C}_{\mathrm{i}}\left(\mathrm{a}_{\mathrm{i}}\right)=\frac{1}{2} \mathrm{c}_{\mathrm{i}} \mathrm{a}_{\mathrm{i}}^{2}$, and $c_{i}$ is salesperson $i$ 's cost coefficient. The problem of the company is:

$$
\begin{aligned}
& \max i m i z e E\left(\pi_{1}+\pi_{2}-S_{1}-S_{2}\right) \\
& \text { s.t. }\left(I R_{i}\right) Y_{i} \geq U_{i} \\
& \left(I C_{i}\right) a_{i} \in \arg \max E\left(S_{i}\right)-\frac{1}{2} \rho \operatorname{Var}\left(S_{i}\right)-C_{i}\left(a_{i}\right)
\end{aligned}
$$

Where $U_{i}$ is the reservation utility of salesperson $I$, (IR) is the participation constraint to ensure the salesman accept this contract, and $\mathrm{IC}_{\mathrm{i}}$ is the incentive compatibility condition, which means the salespersons are to maximize their expected utility. 


\section{Sales personnel compensation contract Compensation}

\subsection{The salesperson's Compensation is linked to both his own performance and his colleague's}

First assume that the compensation of a salesperson are linked to both his own performance and his colleague's. Compensation of the agents is based on a linear function of the two sales staff performance indicators, i.e., $S_{i}=\omega_{i}+\alpha_{i} \pi_{i}+\beta_{i} \pi_{j}$, where $\varpi_{\mathrm{i}}$ is the fix income of sales staff $i, \alpha_{i}, \beta_{i}$ are the incentive compensation index intensity, $\alpha_{\mathrm{i}}$ is the incentive coefficient or proportion that salesperson $i$ shares his own performance and $\beta_{i}$ is the incentive coefficient or proportion that salesperson $i$ shares his colleague' performance, and $\alpha_{i}>0,0<\left|\beta_{i}\right|<\alpha_{i}, i=1,2$.

The principal's problems can be re-expressed as the following:

$\operatorname{maximize} E\left(\pi_{1}+\pi_{2}-S_{1}-S_{2}\right)$

$(I R) \varpi_{i}+\alpha_{i} \pi_{i}+\beta_{i} \pi_{j}-\frac{1}{2} \rho\left(\alpha_{i}^{2} \sigma^{2}+\beta_{i}^{2} \sigma^{2}\right)-\frac{1}{2} c_{i} a_{i}^{2} \geq U_{i}\left(\mathrm{IC}_{1}\right) \mathrm{a}_{1} \in \arg \max \sigma_{1}+\alpha_{1} \sum_{\mathrm{j}=1}^{2} \mathrm{~g}_{1 \mathrm{j}} \mathrm{a}_{\mathrm{j}}+\beta_{1} \sum_{\mathrm{i}=1}^{2} \mathrm{~g}_{2 \mathrm{i}} \mathrm{a}_{\mathrm{i}}-\frac{1}{2} \rho\left(\alpha_{1}^{2} \sigma^{2}+\beta_{1}^{2} \sigma^{2}\right)-\frac{1}{2} \mathrm{c}_{1} \mathrm{a}_{1}^{2}$

$\left(I C_{2}\right) a_{2} \in \arg \max \sigma_{2}+\alpha_{2} \sum_{i=1}^{2} g_{2 i} a_{i}+\beta_{2} \sum_{j=1}^{2} g_{1 j} a_{j}-\frac{1}{2} \rho\left(\alpha_{2}^{2} \sigma^{2}+\beta_{2}^{2} \sigma^{2}\right)-\frac{1}{2} c_{2} a_{2}^{2}$

Incentive compatibility condition $\left(I C_{i}\right)$ can also be expressed as the following first order conditions:

$$
\begin{aligned}
& a_{1}^{*}=\left(\alpha_{1} g_{11}+\beta_{1} g_{21}\right) / c_{1} \\
& a_{2}^{*}=\left(\alpha_{2} g_{22}+\beta_{2} g_{12}\right) / c_{2}
\end{aligned}
$$

Total social output is:

$E\left[\pi^{*}\right]=E\left[\pi_{1}^{*}+\pi_{2}^{*}\right]=\left(g_{11}+g_{21}\right) \frac{1}{c_{1}}\left(\alpha_{1} g_{11}+\beta_{1} g_{21}\right)+\left(g_{12}+g_{22}\right) \frac{1}{c_{2}}\left(\alpha_{2} g_{22}+\beta_{2} g_{12}\right)$

Let conditions IRi take the equal sign and substitute the first-order conditions of ICi into the original objective function, we have:

$$
\max _{\alpha_{1}, \alpha_{2}, \beta_{\mathrm{i}}, \beta_{2}}\left[\sum_{\mathrm{i}=1,1, i, j, j \in(1,2)}^{2}\left(\mathrm{~g}_{\mathrm{iii}}+\mathrm{g}_{\mathrm{ji}}\right) \frac{1}{\mathrm{c}_{\mathrm{i}}}\left(\alpha_{\mathrm{i}} \mathrm{g}_{\mathrm{ii}}+\beta_{\mathrm{i}} \mathrm{g}_{\mathrm{ji}}\right)-\frac{1}{2} \rho\left(\alpha_{\mathrm{i}}^{2} \sigma_{\mathrm{i}}^{2}+\beta_{\mathrm{i}}^{2} \sigma_{\mathrm{j}}^{2}\right)-\frac{\left(\alpha_{\mathrm{i}} \mathrm{g}_{\mathrm{ii}}+\beta_{\mathrm{i}} \mathrm{g}_{\mathrm{ji}}\right)^{2}}{2 \mathrm{c}_{\mathrm{i}}}\right]
$$

Solving for the principal's problem, we get:

$$
\begin{aligned}
& \alpha_{i}^{*}=\frac{g_{i i}\left(g_{i i}+g_{j i}\right)}{\rho \sigma_{i}^{2} c_{i}+g_{i i}^{2}+g_{j i}^{2}} \\
& \beta_{\mathrm{i}}^{*}=\frac{\mathrm{g}_{\mathrm{ji}}\left(\mathrm{g}_{\mathrm{ii}}+\mathrm{g}_{\mathrm{ji}}\right)}{\left(\rho \sigma_{\mathrm{i}}^{2} \mathrm{c}_{\mathrm{i}}+\mathrm{g}_{\mathrm{ii}}^{2}\right)+\mathrm{g}_{\mathrm{ji}}^{2}}
\end{aligned}
$$

It is easy to find:

$$
\alpha_{i}^{*} / \beta_{i}^{*}=g_{i i} / g_{j i}
$$

The above result is based on the compensation function: $S_{i}=\omega_{i}+\alpha_{i} \pi_{i}+\beta_{i} \pi_{j}$.

\subsection{The salesperson's Compensation is only linked to his own sales or performance}

Assume that the compensation of a salesman is only linked to his own performance, i.e., $S_{i}=\omega_{i}+\alpha_{i} \pi_{i}$, and $\alpha_{i}>0, i=1,2$.

The principal's problems can be re-expressed as the following:

$$
\begin{aligned}
& \max \operatorname{imize} E\left(\pi_{1}+\pi_{2}-S_{1}-S_{2}\right) \\
& (I R) \varpi_{i}+\alpha_{i} \pi_{i}+-\frac{1}{2} \rho \alpha_{i}^{2} \sigma^{2}-\frac{1}{2} c_{i} a_{i}^{2} \geq U_{i}
\end{aligned}
$$




$$
\begin{aligned}
& \left(I C_{1}\right) a_{1} \in \arg \max \varpi_{1}+\alpha_{1} \sum_{j=1}^{2} g_{1 j} a_{j}-\frac{1}{2} \rho \alpha_{1}^{2} \sigma^{2}-\frac{1}{2} c_{1} a_{1}^{2} \\
& \left(I C_{2}\right) a_{2} \in \arg \max \varpi_{2}+\alpha_{2} \sum_{i=1}^{2} g_{2 i} a_{i}-\frac{1}{2} \rho \alpha_{2}^{2} \sigma^{2}-\frac{1}{2} c_{2} a_{2}^{2}
\end{aligned}
$$

The incentive compatibility condition $\mathrm{IC}_{\mathrm{i}}$ can be expressed as the first order conditions:

$$
\begin{aligned}
& \mathrm{a}_{1}^{* *}=\frac{\alpha_{1} \mathrm{~g}_{11}}{\mathrm{c}_{1}}, \\
& \mathrm{a}_{2}^{* *}=\frac{\alpha_{2} \mathrm{~g}_{22}}{\mathrm{c}_{2}},
\end{aligned}
$$

With this compensation contract, the total social output is:

$E\left[\pi^{* *}\right]=E\left[\pi_{1}^{* *}+\pi_{2}^{* *}\right]=\frac{1}{c_{1}} \alpha_{1} g^{2}{ }_{11}+\frac{1}{c_{2}} \alpha_{2} g_{22} g_{12}$

There is:

$E\left[\pi^{*}\right]-E\left[\pi^{* *}\right]=\frac{\left(\alpha_{1}+\beta_{1}\right) g_{11} g_{21}+\beta_{1} g_{21}^{2}}{c_{1}}+\frac{\left(\alpha_{2}+\beta_{2}\right) g_{12} g_{22}+\alpha_{2} g_{22}^{2}}{c_{2}}>0$

Therefore, from equation 11, we can see that, when a salesperson's compensation is linked to both his own performance and his colleague's, the social output is greater than that when the compensation is only linked to his own performance, provided that there is a competition relationship between the two salespersons.

\subsection{Factors affecting the incentive degree coefficient}

Since the compensation contract of linking a salesman's compensation to all salespersons' performance is superior to that of linking a salesman's compensation to only his own performance, we choose the former compensation contract for our later analysis.

Since $\alpha_{1}, \alpha_{2}, \beta_{1}, \beta_{2}$ are symmetric, we only need to discuss the factors affecting the incentive coefficient, $\alpha_{1}, \beta_{1}$, for salesperson 1 .

(1)From the first order conditions, we have: $\frac{\partial \alpha_{1}}{\partial \mathrm{c}_{1}}=\frac{-\rho \sigma^{2}\left(\mathrm{~g}_{11}^{2}+\mathrm{g}_{11} \mathrm{~g}_{21}\right)}{\left(\mathrm{g}_{11}^{2}+\mathrm{g}_{21}^{2}+\rho \mathrm{c}_{1} \delta^{2}\right)^{2}}<0, \frac{\partial \beta_{1}}{\partial \mathrm{c}_{1}}=\frac{-\rho \sigma^{2}\left(\mathrm{~g}_{21}^{2}+\mathrm{g}_{11} \mathrm{~g}_{21}\right)}{\left(\mathrm{g}_{11}^{2}+\mathrm{g}_{21}^{2}+\rho \mathrm{c}_{1} \delta^{2}\right)^{2}}>0$. This means that the higher the agent's cost of effort is, the smaller the optimal proportion that the salesperson shares his own performance, and the greater the optimal proportion that the salesperson shares his colleague's performance. This is obvious from our intuitions, the more costly for a salesman to exert effort; the less we should motivate him to do so from the viewpoint of maximizing social output. Since there is a competition relationship between the two salespersons, which means that the salesperson's effort to increase his own performance decreases his colleague's performance, the more costly for him to exert effort, the less that his effort would decrease his colleague's performance, we should motivate him more not to decrease his colleague's performance. Therefore, the more costly for him to exert effort, the more we should tie his compensation to his colleague's performance.

There also is:

$$
\frac{\partial \alpha_{1}}{\partial \rho}=\frac{-c_{1} \sigma^{2}\left(g_{11}^{2}+g_{11} g_{21}\right)}{\left(g_{11}^{2}+g_{21}^{2}+\rho c_{1} \delta^{2}\right)^{2}}<0, \frac{\partial \beta_{1}}{\partial \rho}=\frac{-c_{1} \sigma^{2}\left(g_{21}^{2}+g_{11} g_{21}\right)}{\left(g_{11}^{2}+g_{21}^{2}+\rho c_{1} \delta^{2}\right)^{2}}>0 \text {. This shows that the more the agents is risk averse, the }
$$

smaller the optimal proportion that the salesperson shares his own performance, and the greater the optimal proportion that the salesperson shares his colleague's performance. The reason for this conclusion is primarily the same as the reasons explained above.

There also is: 


$$
\frac{\partial \alpha_{1}}{\partial \delta^{2}}=\frac{-c_{1} \rho\left(g_{11}^{2}+g_{11} g_{21}\right)}{\left(g_{11}^{2}+g_{21}^{2}+\rho c_{1} \delta^{2}\right)^{2}}<0, \frac{\partial \beta_{1}}{\partial \delta^{2}}=\frac{-c_{1} \rho\left(g_{21}^{2}+g_{11} g_{21}\right)}{\left(g_{11}^{2}+g_{21}^{2}+\rho c_{1} \delta^{2}\right)^{2}}<0
$$

This indicates that the more risky the performance,

the smaller the optimal proportion that the salesperson shares his own performance, and the smaller the optimal proportion that the salesperson shares his colleague's performance. The reason for this is straightforward. Since it is less efficient for the agent to share output when the output is more risky, it is better for the agent to share the output, either his own output or his colleague's.

There also is:

$$
\frac{\partial \alpha_{1}}{\partial g_{11}}=\frac{-g_{21} g_{11}^{2}+\left(2 g_{11}+g_{21}\right)\left(g_{21}^{2}+\rho c_{1} \delta^{2}\right)}{\left(g_{11}^{2}+g_{21}^{2}+\rho c_{1} \delta^{2}\right)^{2}}>0 \text {. }
$$

This indicates that the greater the marginal effect of effort of the salesperson, the greater the optimal incentive coefficient is for the salesperson to share his own performance. When the output of the agent's per unit of effort is greater, or the greater the marginal effect of the salesperson's effort, the more efficient per unit of effort is; it is more efficient to motivate the agent to exert effort to improve firm performance, which yields a greater social output.

There also is:

$$
\frac{\partial \alpha_{1}}{\partial g_{21}}=\frac{-g_{21} g_{11}\left(2 g_{11}+g_{21}\right)+g_{11}^{3}+g_{11} \rho c_{1} \sigma^{2}}{\left(g_{11}^{2}+g_{21}^{2}+\rho c_{1} \sigma^{2}\right)^{2}} \text {. When } g_{21}<-g_{11}+\sqrt{2 g_{11}^{2}+\rho c_{1} \sigma^{2}} \text {, or } g_{11}+g_{21}<\sqrt{2 g_{11}^{2}+\rho c_{1} \sigma^{2}}, \quad \partial \alpha_{1} / \partial g_{21}>0 \text {. }
$$

Which means when the effect coefficient that the agent's marginal efforts have on his colleague's performance is less than a certain value minus the marginal effect coefficient that his efforts have on own his performance, or the marginal effect coefficient that the agent's efforts have on his own performance plus the marginal effect coefficient that his efforts have on his colleague's performance is less than a certain value, the greater the marginal effect a salesperson's effort has on his colleague's performance, the greater the proportion that the salesperson should share his own performance. The reason for this is: when $g_{21}$ is smaller than a certain value, the smaller the value of $g_{21}$, the more the colleague's performance is decreased as a result of the salesman's effort to increase his own performance, and the less optimal from the viewpoint of the company; therefore the less we should motivate this salesperson to increase his performance and the smaller the proportion that the salesperson should share his own performance. This can be regarded as a disincentive for the salesperson to harm his colleague's performance.

There also is:

$\frac{\partial \beta_{1}}{\partial g_{11}}=\frac{-g_{21} g_{11}^{2}-2 g_{21}^{2} g_{11}+g_{21}\left(g_{21}^{2}+\rho c_{1} \delta^{2}\right)}{\left(g_{11}^{2}+g_{21}^{2}+\rho c_{1} \delta^{2}\right)^{2}}$. When $g_{11}-g_{21}<\sqrt{2 g_{11}^{2}-\rho c_{1} \sigma^{2}}$, there is $\partial \beta_{1} / \partial g_{11}>0$. That is, the greater the marginal effect of the salesman's effort on his own performance, the greater the proportion that the salesperson should share his colleague's performance, when the difference between the effect coefficient that the agent's efforts have on his own performance and the effect coefficient that his efforts have on his colleague's performance is less than a certain value. The reason is: when the value of $g_{11}-g_{21}$ is smaller than a certain value, the smaller the value of $g_{11}-g_{21}$, the smaller the value of $g_{11}$, given a fixed value of $g_{21}$; the smaller the value of $g_{11}$, given a fixed value of $g_{21}$; the smaller the value of $g_{11}$, the less efficient the salesperson's effort, therefore the less efficient to motivate him to exert effort to improve performance, and it is relatively more efficient to link his compensation to his colleague's performance to discourage him to exert effort.

When $g_{11}-g_{21}>\sqrt{2 g_{11}^{2}-\rho c_{1} \sigma^{2}}$, there is $\partial \beta_{1} / \partial g_{11}<0$. That is, the greater the marginal effect of the salesman's effort on his own performance, the smaller the proportion that the salesperson should share his own colleague's performance, when the difference between the effect coefficient that the agent's efforts have on his own performance and the effect coefficient that his efforts have on his colleague's performance is greater than a certain value. The reason is: when the value of is greater 
than a certain value, the greater the value of $g_{11}-g_{21}$, the greater the value of $g_{11}$, given a fixed value of $g_{21}$; the greater the value of $g_{11}$, the more efficient the salesperson's effort, therefore the more efficient it is to motivate him to exert effort to improve performance, and it is relatively less efficient to link his compensation to his colleague's performance to discourage him to exert effort.

There also is:

$\frac{\partial \beta_{1}}{\partial g_{21}}=\frac{-g_{11} g_{21}^{2}+2\left(g_{11}^{2}+\rho c_{1} \delta^{2}\right) g_{21}+g_{11}^{3}+g_{11} \rho c_{1} \delta^{2}}{\left(g_{11}^{2}+g_{21}^{2}+\rho c_{1} \delta^{2}\right)^{2}}>0$. This indicates that the greater the marginal effect of the salesman's effort on his colleague's performance, the greater the proportion that the salesperson should share his colleague's performance. This is easy to understand: we should motivate the people according to things they have control and the more a task is controlled by a person, the more we should ally that person's profits to the performance of the task.

According to the above equation, or $\alpha_{i}^{*} / \beta_{i}^{*}=g_{i i} / g_{j i}$, we see the value of $g_{i i}$ relative to $g_{j i}$ is equal to the value of $\alpha_{i}$ relative to $\beta_{i}$. One important thing we have not mentioned up to now is that the value of $\beta_{i}$ is always negative, provided that there is a competition relationship between the two agents. The more negative effect a salesperson's effort has on his colleague's performance, the more his compensation is deducted.

\section{Results and conclusions}

Salesperson motivation plays an important role in the sales of firms. Based on the multi-agent principal-agent model set up in the paper, we derive the following conclusions:

First, we should link a salesperson's compensation is to both his own performance and his colleague's, instead of only linking his compensation to his own performance, provided that there is a competition relationship between the two salespersons.

Second, the optimal compensation is always composed of two parts: an incentive for an agent to improve his own performance and a disincentive for the agent to harm his colleague's performance, provided that there is a competition relationship between the two agents. The magnitude of the disincentive depends on the marginal effect of the agent's behavior on his colleague's performance: the more harm his effort does on his colleague's performance, the more his compensation should be deducted.

Third, the higher the agent's cost of effort is, the smaller the optimal proportion that the salesperson shares his own performance; the greater the marginal effect of effort of the salesperson, the greater the optimal incentive coefficient is for the salesperson to share his own performance, when the effect coefficient that the agent's marginal efforts have on his colleague's performance is less than a certain value minus the marginal effect coefficient that his efforts have on own his performance; the greater the marginal effect a salesperson's effort has on his colleague's performance, the greater the proportion that the salesperson should share his own performance.

Last, the greater the marginal effect of the salesman's effort on his own performance, the greater the proportion that the salesperson should share his colleague's performance, when the difference between the effect coefficient that the agent's efforts have on his own performance and the effect coefficient that his efforts have on his colleague's performance is less than a certain value; the greater the marginal effect of the salesman's effort on his own performance, the smaller the proportion that the salesperson should share his colleague's performance, when the difference between the effect coefficient that the agent's efforts have on his own performance and the effect coefficient that his efforts have on his colleague's performance is greater than a certain value; the greater the marginal effect of the salesman's effort on his colleague's performance, the greater the proportion that the salesperson should share his colleague's performance. 


\section{Acknowledgments}

This work was supported by the Ministry of education of Humanities and Social Science project under Grant No. 13YJA630053.

\section{References}

[1] Sunil Dutta, Regina M.Anctil. Exclusive dealing, common agency, and multiprincipal incentive theory[J] . Rand Journal of Economics, 2000, 27: 1 31.

[2] Zhi Xiaoqiang. Analysis on Salesperson Incentive Compensation System based on model of entrust Agency [J]. East China Economic Management.2008, 22(6):98 101

[3] Tian Houping, Liu Changxian, Guo Yajun. Pay Scheme Designing Method with Multiple Agents' competition [J]. Journal of Industrial Engineering/Engineering Management.2009,21(4):153 156.

[4] Tian Ying, Pu Yongjian. The measuring method of human resource value based on real option [J]. Management science and systems science process.2005, 8:394 395.

[5] Lin Yinghui, Tu Meizeng.. Core supply chain enterprise coordination of Incentive Contract Design [J].Shanghai jiaotong university learned journal. 2015, 10:65 69 